\title{
Simultaneous measurement of curvature and strain using a suspended multicore fiber
}

\author{
R. M. Silva, ${ }^{1}$ M. S. Ferreira, ${ }^{1}$ J. Kobelke, ${ }^{2}$ K. Schuster,${ }^{2}$ and O. Frazão ${ }^{1, *}$ \\ ${ }^{1}$ INESC Porto, Rua do Campo Alegre, 687, 4169-007 Porto, Portugal \\ ${ }^{2} I P H T$-Institute of Photonic Technology, D-07745 Jena, Germany \\ ${ }^{*}$ Corresponding author: ofrazao@inescporto.pt
}

Received August 1, 2011; revised September 5, 2011; accepted September 5, 2011; posted September 9, 2011 (Doc. ID 152151); published September 30, 2011

\begin{abstract}
A suspended multicore fiber sensor for simultaneous measurement of curvature and strain is proposed. The spectral response shows evidences of several interferences arising from the seven cores of the fiber. Once the sensing head presents different sensitivities for curvature and strain measurements, these physical parameters can be discriminated by using the matrix method. The $r m s$ deviations are $\pm 19 \mathrm{~m}^{-1}$ and $\pm 12.90 \mu \varepsilon$ for curvature and strain measurements, respectively. (C) 2011 Optical Society of America

OCIS codes: $\quad 060.0060,060.2310,060.2370,060.4005$.
\end{abstract}

Multicore fibers, first introduced by Scifres [1] in 1996, have been mainly investigated in lasing and passive optical networks. Several applications have been proposed, such as switching, phase-locked high-power lasers [2] and dividing/combining the optical power in optical fiber networks [3].

In 2002, Araújo et al. firstly reported a miniaturized curvature, curvature plane, and temperature sensor configuration based on a triangular geometry. This comprised three single-mode fiber sections with fiber Bragg gratings (FBGs) inscribed in them [4]]. A two-axes curvature sensor with FBG multicore optical fiber was presented by Flockhart et al. The sensor measured both magnitude and plane of curvature, being temperature independent [5]. In 2005, Mafi et al. reported a formalism that provided a simple recipe to modify and customize mode shapes for multicore photonic crystal fibers (PCFs) [2]. Two years later, a phase-locked multicore fiber laser with multiple cores arranged in a rectangular array was proposed by Zhu et al. [6] . They measured the birefringence during in-phase mode operation of a multicore fiber laser for the first time. Varshney et al. investigated a new design of a multicore PCF that was able to divide a single optical power into four ports with $6 \mathrm{~dB}$ of power in each core [3]. In 2010, Zhu et al. reported the use of a seven-core multicore fiber for passive optical network. In this case, the seven cores were arranged in a hexagonal array [7]. A multicore design strategy for obtaining single polarization in-phase supermode operation was proposed by Wang et al. [8].

Recently, some works using suspended core fibers have been reported in the sensing field. A Fabry-Pérot sensing structure based on a one-core suspended fiber was demonstrated [9]. The sensing head was able to perform strain and temperature measurements. An all fiber Mach-Zehnder interferometer using a suspended twincore fiber as a sensing element was also reported. In this case, the sensing head was subjected to curvature and temperature. With this kind of fiber it was possible to discriminate the measurands [10]. A torsion sensor based on a fiber loop mirror configuration using a suspended twin-core fiber was also presented. The sensing element exhibited strain and temperature independence [11].
In this Letter, a configuration based on a suspended multicore fiber is proposed. Because of the interference of light that propagates in the seven cores, it is possible to perform simultaneous measurement of curvature and strain.

The experimental setup is presented in Fig. 1(a)]. A broadband optical source with a bandwidth of $100 \mathrm{~nm}$ and a central wavelength at $1550 \mathrm{~nm}$ was used to inject light in the interferometers. An optical spectrum analyzer (OSA) was used to measure the transmission spectral response of the sensing head with a resolution of $0.1 \mathrm{~nm}$. The sensing head was constituted by a seven suspended core fiber section spliced between two small sections of light expansible fiber. These sections were, in their turn, spliced to a section of single-mode fiber (SMF), according to the scheme presented in Fig. 1(b)].

The light expansible fiber section, based on graded index fiber (GIF) had a length of $\sim 530 \mu \mathrm{m}$. This value was obtained according to[12] and corresponded to the presence of a light antinode at the end of the GIF section.

(a)

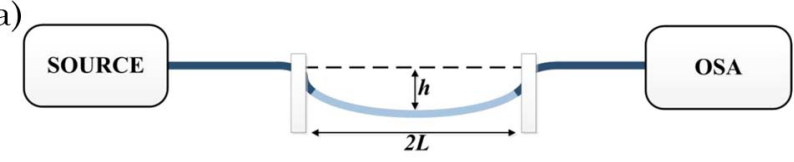

(b)

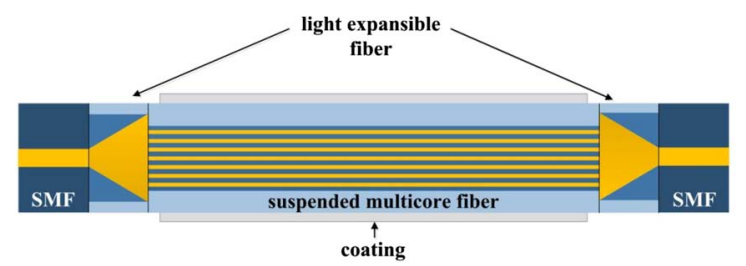

(c)
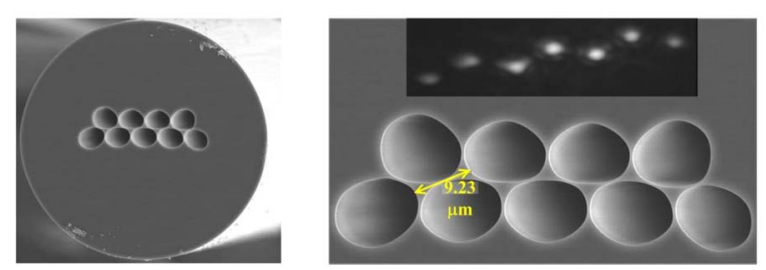

Fig. 1. (Color online) (a) Experimental setup scheme. (b) Detail of the sensing head composition. (c) Cross-section of the suspended multicore fiber, where the nine holes are visible. Inset: illumination of the seven cores. 
Thus, it was expected that the suspended multiple core fiber would be better illuminated and light would travel along the multiple cores, producing high interferences. Figure 1(c)shows the cross section of the suspended multicore fiber, which was fabricated at the Institute of Photonic Technology (Jena, Germany). It presents nine air holes made of pure silica and in between them there are seven triangular cores, as can be seen in the inset Fig. 1(c), where they were directly illuminated. The distance between the cores is $\sim 9.23 \mu \mathrm{m}$. The sensing head had a length of $25.3 \mathrm{~cm}$. The suspended multicore fiber coating played an important role, since it absorbed the light travelling in the cladding. This guaranteed that all interference occurred due to the light propagation inside the cores. In a general way, the interference depends upon the optical path length difference between the interferometer arms and the effective indices difference between the cores. In this configuration, the optical paths are the same, therefore the interference occurs due to the refractive index difference between the cores. This is due to the difference of stress geometry of the cores.

The transmission spectral response can be observed in Fig. 2. The spectrum has a complex appearance and several beats can be distinguished. The inset in Fig. 2 clearly shows the multiple fringes inside the larger beat, which has a $\sim 10 \mathrm{~nm}$ period. These fringes arise from the interference between the different cores. Figure 3 presents the fast Fourier transform (FFT), where multiple frequencies can be identified, some with higher amplitude than others.

The refractive index difference was calculated for the three strongest peaks, located at frequencies of $0.09 \# / \mathrm{nm}, 0.28 \# / \mathrm{nm}$, and $584 \# / \mathrm{nm}$ and values of $1 \times 10^{-3}, 16 \times 10^{-3}$, and $66 \times 10^{-3}$ RIU (refractive index unit) were respectively obtained.

Curvature measurements were performed by placing the sensing head between two clamps, whose halfdistance between edges was $L$. The curvature radius, $r$, is given by $1 / r=2 h /\left(h^{2}+L^{2}\right)$, where $h$ is the bending displacement at the center of the suspended multicore fiber. The measurements were done guaranteeing that

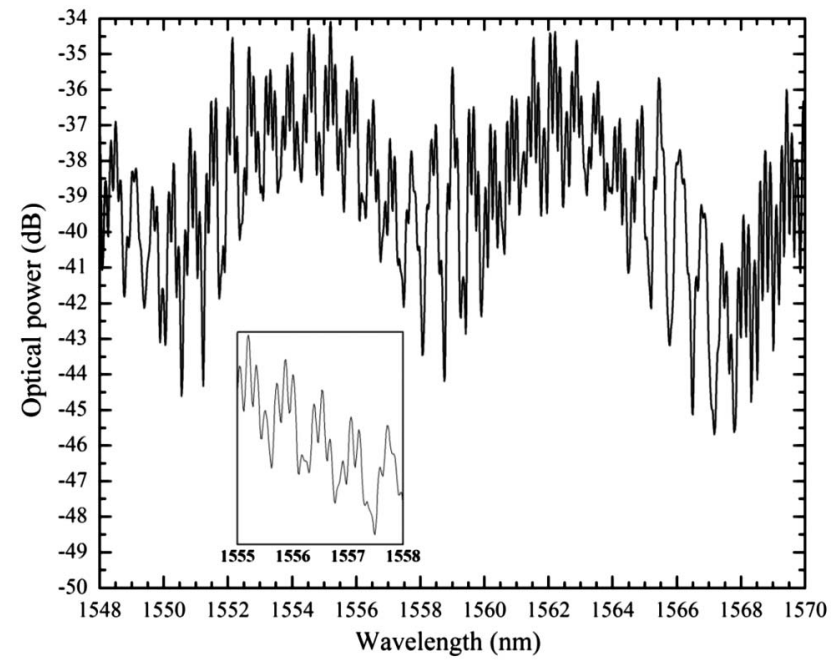

Fig. 2. Spectral response of the sensing head. Inset: detail of the spectral response for a small wavelength range.

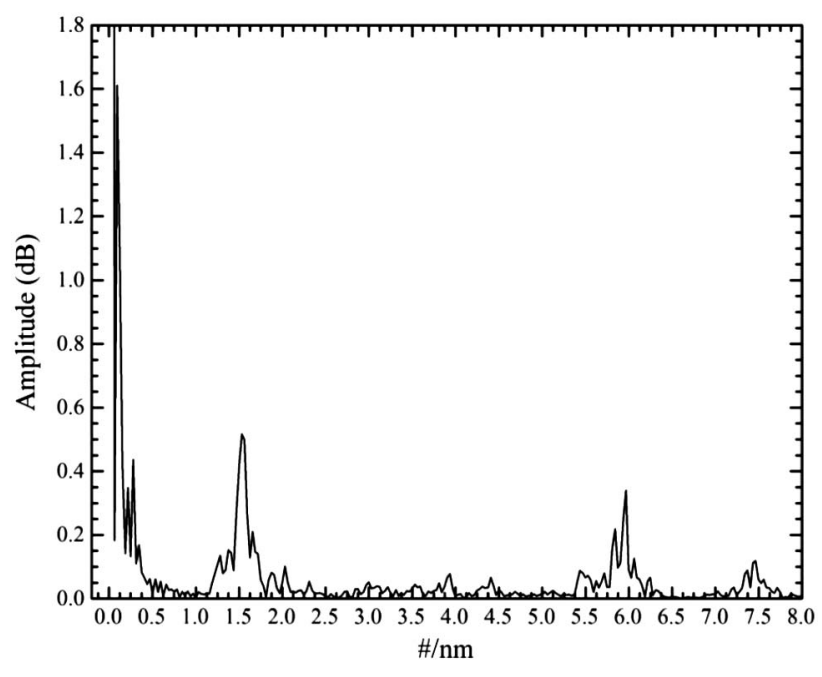

Fig. 3. Spatial frequency spectrum.

the core array was perpendicularly oriented in relation to the plane of the bend.

Figure 4 shows the amplitude behavior, determined through the FFTs, towards the curvature radius. Two distinct beat frequencies were analyzed, at $0.09 \# / \mathrm{nm}$ and $0.28 \# / \mathrm{nm}$. Even though the frequencies are relatively close to each other, their behavior in respect to curvature is quite different. The opposite response occurs due to the compression of some cores, while others are in tension.

This results in an optical path change in respect to the central core in the neutral zone. The different response is due to the asymmetric geometry of the fiber cores. The inset in Fig. 4 shows the linear regions $\left(0.25-0.45 \mathrm{~m}^{-1}\right)$ of these curves, from which sensitivities of $-4.57 \mathrm{dBm}$ and $1.29 \mathrm{dBm}$ were obtained for the smaller and larger frequency, respectively.

Regarding strain measurements, the sensing head was attached to a translation stage with a resolution of $1 \mu \mathrm{m}$. All measurements were performed at room temperature $\left(\sim 23^{\circ} \mathrm{C}\right)$ and the same frequencies were analyzed. For this measurand, the two beats have also different behaviors; while the beat with larger frequency presents a negative sensitivity, $-1.5 \times 10^{-3} \mathrm{~dB} / \mu \varepsilon$, the other beat analyzed shows a positive sensitivity, $7.6 \times 10^{-3} \mathrm{~dB} / \mu \varepsilon$.

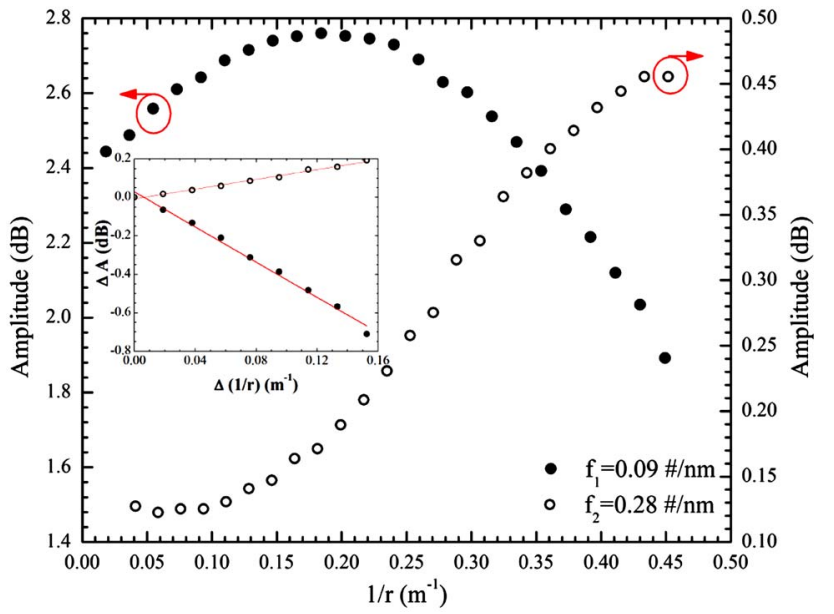

Fig. 4. (Color online) Sensing head response when curvature was applied. 


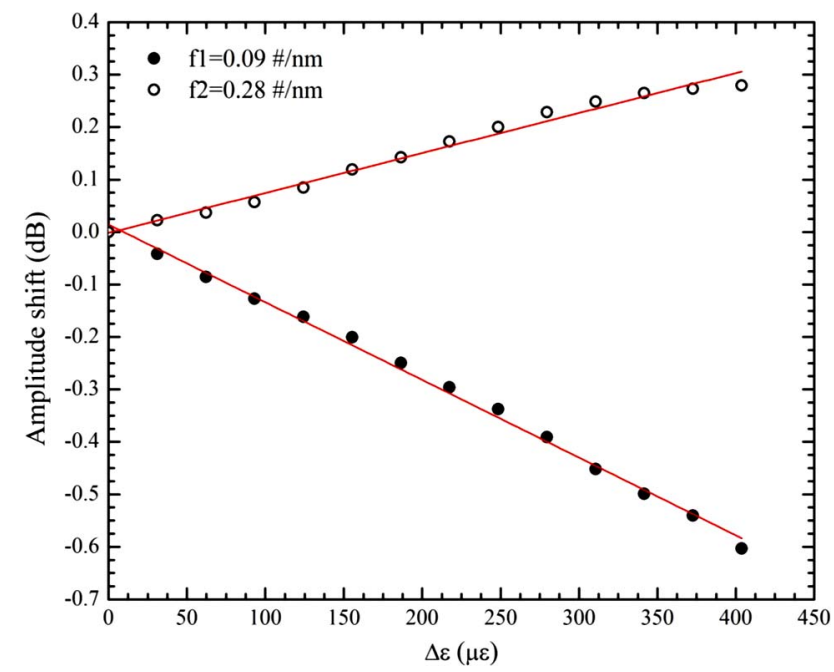

Fig. 5. (Color online) Response of the sensing head when longitudinal strain is applied. (No curvature was applied.)

See Fig. 5. This opposite behavior can be explained due to the increase of the optical path length of one of the interferometers, resulting in a wavelength increase (red-shift). The other interferometer presents a negative response because the refractive index variation, attributed to the silica thermooptic effect, is dominant.

The observed difference in the sensitivity coefficients allows the possibility of using this sensing head for simultaneous measurements of curvature and strain. Thus, the following matrix can be written:

$$
\left[\begin{array}{c}
\Delta \varepsilon \\
\Delta C
\end{array}\right]=\frac{1}{D}\left[\begin{array}{cc}
k_{C 2} & -k_{C 1} \\
-k_{\varepsilon 2} & k_{\varepsilon 1}
\end{array}\right]\left[\begin{array}{l}
\Delta A 1 \\
\Delta A 2
\end{array}\right]
$$

where $D=k_{C 2} k_{\varepsilon 1}-k_{C 1} k_{\varepsilon 2}$ is the matrix determinant, $\Delta C$ corresponds to $1 / r$ and $\Delta A$ is the amplitude shift. The $\Delta A, \Delta C$, and $\Delta \varepsilon$ are given in $\mathrm{dB}$ (decibel), $\mathrm{m}$ (meter) and $\mu \varepsilon$ (microstrain), respectively. Inserting the sensitivity coefficients calculated previously yields:

$$
\left[\begin{array}{c}
\Delta \varepsilon \\
\Delta C
\end{array}\right]=\frac{1}{1.54 \times 10^{-3}}\left[\begin{array}{cc}
1.29 & 4.57 \\
-7.60 \times 10^{-4} & 1.50 \times 10^{-3}
\end{array}\right]\left[\begin{array}{c}
\Delta A 1 \\
\Delta A 2
\end{array}\right] .
$$

The rms deviations for curvature and strain measurements were $\pm 0.19 \mathrm{~m}^{-1}$ and $\pm 12.90 \mu \varepsilon$, respectively.
For the temperature measurements, the sensing head was placed in a tubular oven and the temperature reading had a resolution of $0.1^{\circ} \mathrm{C}$. The fringe amplitude presented very low dependence with temperature when the FFT analysis was used. This behavior is similar to the one reported in the literature [11].

In summary, a suspended multicore fiber sensor for simultaneous measurement of curvature and strain was demonstrated. Light that travelled inside the seven cores interfered and gave rise to a complex spectrum. Curvature and strain measurements were done by analyzing the FFT amplitude shift for two different frequencies. The lower frequency was the most sensitive, for both measurements. The several frequencies observed in the transmission spectrum have different responses to the applied measurands. If a good coupling/recoupling between the SMF core and the suspended multicore fiber is guaranteed, it is possible to use this sensing head for multiparameter discrimination.

\section{References}

1. D. R. Scifres, "Multiple core fiber laser and optical amplifier," U.S. patent 5,566,196 (1994).

2. A. Mafi and J. V. Moloney, IEEE Photon. Technol. Lett. 17, 348 (2005).

3. S. K. Varshney, K. Saitoh, R. K. Sinha, and M. Koshiba, J. Lightwave Technol. 27, 2062 (2009).

4. F. M. Araújo, L. A. Ferreira, and J. L. Santos, Appl. Opt. 41, 2401 (2002).

5. G. M. H. Flockhart, W. N. MacPherson, J. S. Barton, J. D. C. Jones, L. Zhang, and I. Bennion, Opt. Lett. 28, 387 (2003)

6. X. Zhu, L. Li, H. Li, V. L. Temyanko, J. V. Moloney, and N. Peyghambarian, Opt. Express 15, 10340 (2007).

7. B. Zhu, T. F. Taunay, M. F. Yan, J. M. Fini, M. Fishteyn, E. M. Monberg, and F. V. Dimarcello, Opt. Express 18, 11117 (2010).

8. C. Wang, L. Gong, J. Li, F. Zhang, and S. Jian, Opt. Commun. 284, 4215 (2011).

9. O. Frazão, S. H. Aref, J. M. Baptista, J. L. Santos, H. Latifi, F. Farahi, J. Kobelke, and K. Schuster, IEEE Photon. Technol. Lett. 21, 1229 (2009).

10. O. Frazão, S. F. O. Silva, J. Viegas, J. M. Baptista, J. L. Santos, J. Kobelke, and K. Schuster, IEEE Photon. Technol. Lett. 22, 1300 (2010).

11. O. Frazão, R. M. Silva, J. Kobelke, and K. Schuster, Opt. Lett. 35, 2777 (2010).

12. Y. Gong, Y. Guo, Y. Rao, T. Zhao, and Y. Wu, IEEE Photon. Technol. Lett. 22, 1708 (2010). 